\title{
Gallstone ileus: Criteria for preoperative diagnosis
}

\author{
VICTOR J. SOBOLEWSKI, III, D.O. \\ Woodbury, New Jersey
}

Gallstone ileus is a difficult diagnosis to reach preoperatively. However, several criteria can aid in diagnosis: (1) history of previous gallbladder symptomatology; (2) lack of previous abdominal surgery; (3) age older than 65 years; (4) female sex; (5) and/or roentgenographic evidence of pneumobilia, two distinct right upper abdominal quadrant air fluid levels, small bowel obstruction, or any radiopaque density resembling a gallstone. In the case reported, some of these factors were present, yet the diagnosis of gallstone ileus was not entertained preoperatively. Because of coexistent medical conditions in the majority of patients, delayed diagnosis may lead to significantly increased morbidity and mortality. Thus, awareness of and suspicion for this disorder become of paramount importance. Only a minimal delay should be allowed for rehydration and electrolyte imbalance correction before enterolithotomy and gallstone removal, with or without cholecystectomy.

With a lack of obvious roentgenographic findings in gallstone ileus, preoperative diagnosis can be very difficult. A high index of suspicion is necessary to recognize this entity in a scenario of typical small bowel obstruction. Gallstone ileus represents only 1 to 3 per cent of all small bowel obstructions, but the incidence rises to 25 percent in patients aged 65 years or older. ${ }^{1}$ The average age approaches 74 years, and a female predominance ranging from $3: 1$ to $9: 1$ has been reported from a large number of studies. ${ }^{1}$ Preoperative diagnosis has been correct in only about 40 percent of cases, and, short of radiographic findings, only a history of previous gallbladder symptomatology has been found to be helpful.

It is the purpose of this case report and review to illustrate and emphasize all of the salient factors that can be used preoperatively to aid in recognition of gallstone ileus. Morbidity, particularly wound infections, ranges from 11-75 percent, and mortality ranges from $8-20$ percent. ${ }^{2,3}$ Prompt diagnosis is critical, since several studies have shown a risked increase in morbidity and mortality if there is an operative delay. ${ }^{2,4}$ More recently, controversy has arisen as to whether perioperative antibiotics are helpful in decreasing morbidity and mortality. 1,3

\section{Report of case}

A 73-year-old white woman was admitted with a 4-day history of progressively increasing abdominal pain and distention. She complained of colicky periumbilical pain, which began suddenly after she drank one glass of eggnog. Immediately thereafter, she experienced several bouts of nausea and vomiting with epigastric abdominal pain, which continued to increase in intensity until admission. She had no oral intake or bowel movement after drinking the beverage. There was no prior history of a similar event, and the remainder of her gastrointestinal history was unremarkable.

The medical history revealed a seizure disorder of 50 years' duration, which was controlled with phenobarbital, and a 3-year history of diet-controlled hypertension. She denied any previous surgery or allergies.

Physical examination revealed a tachycardic, tachypneic, elderly woman in acute abdominal distress. Her oral temperature was $99.4 \mathrm{~F}$, and her blood pressure was $150 / 90 \mathrm{~mm}$. $\mathrm{Hg}$. The mucous membranes and axillae were dry, and the skin turgor was poor. The abdomen was distended, tympanitic, and diffusely tender to palpation. Peristalsis was active and high pitched. No abdominal 
scars were observed; however, there was a fascial deficiency in the abdominal wall paraumbilically. The rectal examination was essentially normal, with no stool found in the ampulla.

Initial laboratory findings revealed the following values: leukocyte count, 13,800/cu.mm. (normal, 4,500-11,000/cu. mm.); hemoglobin, $12.4 \mathrm{gm} . / \mathrm{dl}$. (normal, 12-16 gm./dl.); serum sodium, $133 \mathrm{mEq} . / \mathrm{L}$. (normal, 135-150 mEq./L.); serum potassium, $3.6 \mathrm{mEq} . / \mathrm{L}$. (normal, 3.5-5.5 mEq./L.); blood urea nitrogen, $64 \mathrm{mg} . / \mathrm{dl}$. (normal, 7-24 mg./dl.); serum creatinine, $2.0 \mathrm{mg} . / \mathrm{dl}$. (normal, 0.6-1.5 mg./dl.); serum alkaline phosphatase, 35 I.U./dl. (normal, 5.0-13.6 I.U./dl.); and total serum bilirubin, 2.4 mg./dl. (normal, 0.1-1.5 mg./dl.).

Radiography of the abdomen revealed a stepladder small bowel obstruction, which appeared complete, as well as a $2-\mathrm{cm}$. radiopaque density in the lower right quadrant, which was thought to be a phlebolith.

Upon insertion of a nasogastric tube, feculent material was secured, and the patient's pain was lessened momentarily. Emergency laparotomy was then performed. At surgery, a right paramedian incision was made, and complete obstruction of the ileum in its midportion was found to be produced by a calculus measuring approximately 4 $\times 6 \mathrm{~cm}$. The intestine distal to this obstruction was collapsed, and the proximal intestine was extremely dilated and contained semifeculent fluid. Enterolithotomy was performed, and the calculus was removed. A thorough search for other calculi within the intestine had negative results. Intestinal closure was attained using a Connell-type suture utilizing 000 Vicryl followed by a 000 Ethibond seromuscular plicating layer.

At this point, because of the dense adhesive process found surrounding the gallbladder, it was decided that a right subcostal incision would be necessary to facilitate appropriate visualization and palpation of the gallbladder and surrounding structures. Thus, closure of the paramedian incision was completed using interrupted sutures of 000 Vicryl on the fascial layer and 00 silk on the skin. After the right subcostal incision was made, lysis of some of the adhesions was performed. The gallbladder was observed to be very contracted, with no evidence of residual calculi in the gallbladder, the cystic duct, or in any of the remaining biliary ducts. Dense adhesions matted the duodenum and proximal transverse colon together. It was thought that any further attempt to mobilize the intestine would risk creating a perforation or tearing significant liver substance, and, therefore, no other surgical manipulation was performed. The right subcostal incision was closed in the same fashion as the paramedian incision.

The patient's postoperative recovery was uneventful except for minimal atelectasis, which responded to aggressive pulmonary toilet. The nasogastric tube was removed on the fourth postoperative day, and the patient's diet was advanced without incident. All laboratory studies were determined to be within normal range prior to her discharge on the ninth postsurgical day. Abdominal roentgenography revealed resolution of the small bowel obstruction, a suggestion of pneumobilia, and the same phlebolith in the lower right quadrant. At her 6-month follow-up visit, the patient felt well, and, because she had no gallbladder symptomatology, no further studies were planned.

\section{Discussion}

Gallstone ileus is a mechanical small bowel obstruction that is caused by impaction of one or more gallstones within the lumen. It is preceded by an initial episode of cholecystitis that results in adhesions between the gallbladder and portions of intestine immediately adjacent to it. One or more gallstones erode through the joined walls to form a cholecystoenteric fistula. ${ }^{1}$ In more than 50 percent of the cases this is located in the duodenum, but it may involve the stomach, colon, or multiple sites..$^{2,5}$

Once the gallstone(s) pass(es) into the intestine, it/they can be expulsed by vomiting or passed spontaneously (80-90 percent), ${ }^{1}$ or it/they can impact in the small intestine. Generally, gallstones smaller than $2.0 \mathrm{~cm}$. pass, while larger ones result in obstruction. The site of the blockage usually is the terminal ileum, which is thought to be both the narrowest segment and the area where peristalsis is the weakest. With recurrent bouts of incomplete obstruction, a "tumbling" phenomenon can occur. It is described as such because symptoms are often intermittent as the stone obstructs, becomes free, and moves distally when reobstruction occurs. ${ }^{3}$ This can be puzzling to the physician and makes diagnosis more obscure.

\section{Diagnosis}

Physical examination commonly reveals only the findings of small bowel obstruction. The patient typically is acutely ill and dehydrated, and he/she has abdominal pain and distention with bowel sounds of obstructive quality. Right upper quadrant tenderness in the vicinity of the gallbladder may indicate acute cholecystitis. Laboratory data reflect the state of dehydration and electrolyte imbalance. Usually, there are only minor abnormalities in liver function values or serum bilirubin levels, and the leukocyte count is only mildly elevated.

Roentgenographic findings are suggestive but not diagnostic. In 1941, Rigler and associates ${ }^{4}$ described the four classic radiographic findings in gallstone ileus - air in the biliary tract, partial or complete small bowel obstruction, direct or indirect visualization (utilizing contrast medium) of the obstructing stone, and a positional change of a previously observed stone.

Pneumobilia can be a consequence of other entities, such as previous biliary tract surgery or an incompetent sphincter of Oddi. However, with signs of intestinal obstruction, and no previous pertinent 
history, gallstone ileus becomes more likely. Review of the literature reveals that defining air in the biliary tree is extremely difficult and only presumptive. In a large study 6 involving radiographic analysis of patients with gallstone ileus, two distinct air fluid levels in the right upper quadrant were found to represent the duodenal cap adjacent to the shrunken gallbladder. Because air fluid levels in this area are infrequent and seen only in paralytic ileus and proximal intestinal obstructions, this finding is suggestive of a cholecystoenteric fistula. Air in the biliary radicles themselves is found only rarely. ${ }^{6}$ Therefore, without the finding of two distinct air fluid levels in the right upper quadrant or biliary tree, only the visualization of a gallstone (15-20 percent of cases) can lead to a preoperative diagnosis via roentgenography. ${ }^{6}$

Eighty percent of patients with gallstone ileus also have serious concomitant disease, including diabetes mellitus and cardiac and pulmonary disease. ${ }^{5}$ Because of this fact, gallstone ileus carries five times the mortality rate of any other nonmalignant cause of small bowel obstruction. It is also for the reason that prompt diagnosis of gallstone ileus is necessary.

\section{Treatment}

The treatment of choice for gallstone ileus is surgery to remove the offending stone(s). Only a short delay for rehydrating the patient and correcting any electrolyte imbalance should be allowed. Nineteen forty-one statistics ${ }^{4}$ revealed a staggering increase in mortality, from 45 percent to 87 percent if a delay of $>72$ hours was allowed. Today, mortality has declined significantly; however, with increased coexistent medical conditions and the length of time of the surgery performed, delay in treatment is risky. Postoperative wound infection is the most common complication and has led to death. ${ }^{3}$ Antibiotic prophylaxis has been shown to be beneficial in one study, ${ }^{6}$ and of no benefit in another. ${ }^{1}$

The operation itself involves longitudinal enterolithotomy and removal of the gallstone(s) by milking it/them to an uninvolved segment of intestine. If it/they cannot be moved into healthy intestine, segmental bowel resection may be required. It should be noted that manipulating the stone(s) can cause subserosal rupture. Following removal, the enterotomy is closed in a transverse fashion. Before closure, a thorough search for additional stones must be made, because some authorities relate an incidence of multiple stones of 10-44 percent, ${ }^{1}$ and a 5 -percent reobstruction rate. ${ }^{2} \mathrm{Also}$, the gallbladder and the cholecystenteric fistula must be checked for evidence of leakage.

A one-stage procedure that includes cholecystec- tomy is advocated by many surgeons, because there is a 15-percent risk of gallbladder carcinoma with cholecystoenteric fistula versus a 1-percent risk without a fistula as well as a 30 -percent incidence of recurrent pain postoperatively when enterolithotomy is performed alone. ${ }^{1}$ It must be emphasized that careful selection of patients is necessary, because most patients may not be able to tolerate the extensive surgery involved. Also, the amount of dissection and associated level of surgical expertise needed must be considered.

Fistulas usually close spontaneously, with the majority of patients never having further symptoms. If cholecystectomy is not performed initially, upper gastrointestinal studies may be carried out postoperatively to demonstrate gallstones, regardless of whether the patient has symptoms. If gallstones are present, elective cholecystectomy may be scheduled. However, there is controversy as to whether further studies are needed for patients who had no additional stones palpated at the time of original surgery and who lack symptoms postoperatively. The complication rate from surgery ranges $8-78$ percent, ${ }^{2-4}$ with the majority of complications relating to wound infections. As stated previously, the role of perioperative antibiotics is controversial.

\section{Comment}

Studies have shown that over the years, there has been no improvement in the diagnostic ability of surgeons faced with gallstone ileus. ${ }^{5}$ Although the length of hospital stay has increased, the mortality rate has remained constant at $10-15$ percent overall. ${ }^{5}$ The mortality rate increases with age, and it reaches 42 percent in patients who are older than 73 years. $^{1}$

The reported case illustrates the need for a high index of suspicion in an elderly female patient who presents with acute bowel obstruction. Although gallstone ileus is an uncommon disorder, the surgeon must remain aware of its occurrence because of the serious consequences of delay in treatment.

\footnotetext{
1. Kurtz, R.J., Heimann, T.M., and Kurtz, A.B.: Gallstone ileus. A diagnostic problem. Am J Surg 146:314-7, Sep 83

2. Van Landingham, S.B., and Broders, C.W.: Gallstone ileus. Surg Clin North Am 62:241-7, Apr 82

3. Deitz, D.M., et al.: Improving the outcome in gallstone ileus. Am J Surg 151:572-6, May 86

4. Rigler, L.G., Borman, C.N., and Noble, J.F.: Gallstone obstruction. Pathogenesis and roentgen manifestations. JAMA 117:1753-9, 22 Nov 41 5. Kurtz, R.J., et al.: Patterns of treatment of gallstone ileus over a 45 year period. Am J Gastroenterol 80:95-8, Feb 85

6. Balthazar, E.J., and Schecter, L.S.: Air in the gallbladder. A frequent finding in gallstone ileus. Am J Roentgenol 131:219-22, Aug 78

Schwartz, S.I., et al.: Gallbladder and extrahepatic biliary system. In Principles of surgery, edited by S.I. Schwartz. Ed. 3. McGraw-Hill Book Company, New York, 1979, pp. 1332-33
} 
Accepted for publication in August 1986. Updating as necessary, has been done by the author.

At the time this paper was written, Dr. Sobolewski was a general surgery resident at Delaware Valley Medical Center, Lan- ghorne, Pennsylvania. Leonard Segal, D.O., is the general surgery residency program director. Dr. Sobolewski is now in family practice and sports medicine in Woodbury, New Jersey.

Dr. Sobolewski, 1000 Mantua Pike, Woodbury, New Jersey 08096. 